\title{
Zn-Catalyzed Asymmetric Allylation for the Synthesis of Optically Active Allylglycine Derivatives. Regio- and Stereoselective Formal $\alpha$-Addition of Allylboronates to Hydrazono Esters
}

\author{
Mari Fujita, Takashi Nagano, Uwe Schneider, Tomoaki Hamada, \\ Chikako Ogawa, and Shū Kobayashi* \\ Department of Chemistry, School of Science and Graduate School of \\ Pharmaceutical Sciences, The University of Tokyo, The HFRE Division, \\ ERATO, Japan Science Technology Agency (JST), Hongo, Bunkyo-ku, \\ Tokyo 113-0033, Japan
}

\section{Supporting Information}

Experimental

S-1

Preparation of a $\mathrm{ZnF}_{2}$ Stock Solution

S-2

Typical Procedure for Asymmetric Allylation with Allylboronates 2 S-2

$\mathrm{Zn}(\mathrm{OH})_{2}$-Catalyzed Asymmetric Allylation $\quad$ S-4

NMR Experiments for Allylzinc Species $\quad$ S-5

Determination of the Absolute Configuration of Compound 3m S-6

References

S-7

Copies of ${ }^{1} \mathrm{H}$ and ${ }^{13} \mathrm{C}$ NMR Spectra of Products 3h-m

S-8

\section{Experimental}

General:

${ }^{1} \mathrm{H}$ and ${ }^{13} \mathrm{C}$ NMR spectra were recorded on a JEOL JNM-ECX400 and JNM-ECX600 spectrometer. Tetramethylsilane (TMS) served as internal standard $(\delta$ $=0 \mathrm{ppm})$ for ${ }^{1} \mathrm{H} \mathrm{NMR}$, and $\mathrm{CDCl}_{3}$ was used as internal standard $(\delta=77.0 \mathrm{ppm})$ for ${ }^{13} \mathrm{C}$ NMR. IR spectra were measured using a JASCO FT/IR-610 spectrometer. Optical rotations were measured with a JASCO P-1010 polarimeter. High resolution ESI mass spectrometry was carried out using BRUKER DALTONICS BioTOF II. High-performance liquid chromatography was carried out using the following apparatus: SHIMADZU LC-10AT (liquid chromatograph), SHIMADZU SPD-10A (UV detector), and SHIMADZU C-R6A Chromatopac. ICP analysis was performed on SHIMADZU ICPS-7510. Preparative thin-layer chromatography was carried out using Wakogel B-5F.

$\mathrm{ZnF}_{2}$ hydrate and $\mathrm{Zn}(\mathrm{OH})_{2}$ were purchased from Aldrich Co., Inc. and Soekawa Rikagaku Co., LTD., respectively. Water was supplied from MILLIPORE Gradient A10 and acetone was purchased from Kanto Kagaku CO., LTD. Chiral Diamines 4 were prepared according to literature procedure. ${ }^{1}$ 
Methyl [ $N^{\prime}$-(p-dimethylaminobenzoyl)hydrazono]-acetate (1h):

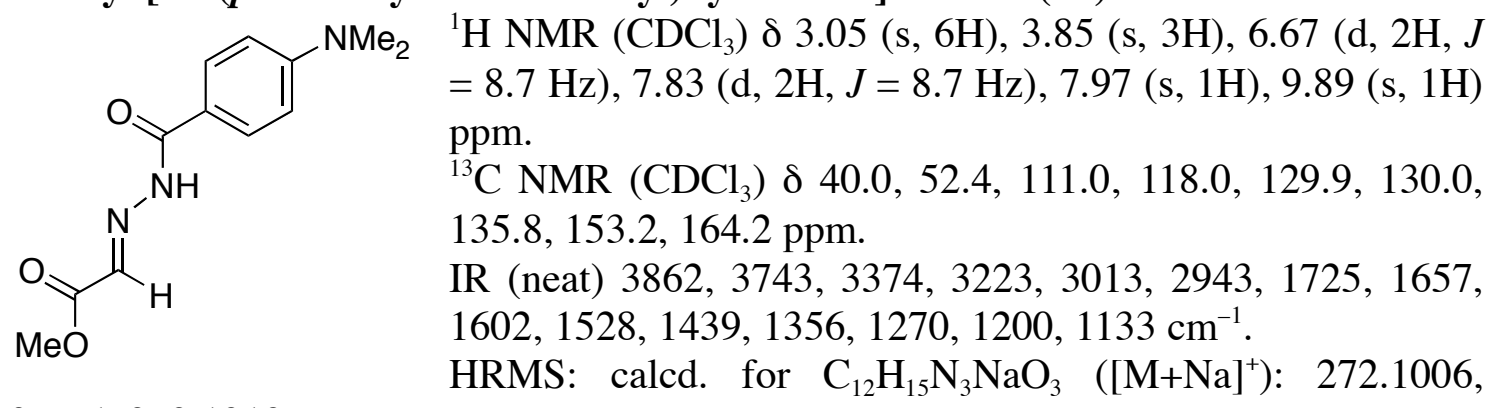

found: 272.1013 .

\section{Preparation of a $\mathbf{Z n F}_{2}$ Stock Solution:}

$\mathrm{ZnF}_{2}$ hydrate $(319.0 \mathrm{mg})$ was added to water $(300 \mathrm{~mL})$, and the mixture was stirred for $5 \mathrm{~min}$. After sonication $(10 \mathrm{~min})$, the solution was filtrated with Kiriyama filtration paper (No. 4 and No. 5B). The concentration of this solution was determined by ICP analysis, and the solution was stocked at $4{ }^{\circ} \mathrm{C}$.

\section{Typical Procedure for Asymmetric Allylation with Allylboronates 2:}

A $\mathrm{ZnF}_{2}$ stock solution $(0.159 \mathrm{mM}, 1.86 \mathrm{~mL}, 0.015 \mathrm{mmol})$ was diluted with water $(0.36 \mathrm{~mL})$, and then $\mathbf{4} \mathbf{a}^{1}(16.2 \mathrm{mg}, 0.036 \mathrm{mmol})$ and acetone $(3.75 \mathrm{~mL})$ were added, and the mixture was stirred for $30 \mathrm{~min}$. After addition of $\mathbf{1 h}(75.0 \mathrm{mg}, 0.30 \mathrm{mmol})$, the mixture was stirred at $0{ }^{\circ} \mathrm{C}$ for $20 \mathrm{~min}$, and then $2 \mathbf{a}(69 \mu \mathrm{L}, 0.36 \mathrm{mmol})$ was added. After $36 \mathrm{~h}$, the reaction was quenched with saturated aqueous $\mathrm{NaHCO}_{3}$. The resultant mixture was extracted with $\mathrm{CH}_{2} \mathrm{Cl}_{2}$ (4 times), and the combined organic layers were dried over anhydrous $\mathrm{Na}_{2} \mathrm{SO}_{4}$. The solvents were evaporated in vacuo, and the residue was purified by preparative TLC $($ Hexane/AcOEt $=1 / 4)$ to give $\mathbf{3 h}$ (quantitative yield). The enantiomeric excess of the product was determined by HPLC analysis (90\% ee).

(R)-Methyl 2-[N'-(p-dimethylaminobenzoyl)hydrazino]-4-pentenoate (3h):<smiles>C=CCC(NNC(=O)c1ccc(N(C)C)cc1)C(=O)OC</smiles>

${ }^{1} \mathrm{H}$ NMR $\left(\mathrm{CDCl}_{3}\right) \delta 2.43-2.51(\mathrm{~m}, 1 \mathrm{H}), 2.59-2.65(\mathrm{~m}, 1 \mathrm{H})$, $3.01(\mathrm{~s}, 6 \mathrm{H}), 3.75(\mathrm{~s}, 3 \mathrm{H}), 3.85(\mathrm{~m}, 1 \mathrm{H}), 5.09$ (brs, $1 \mathrm{H}), 5.18$ $(\mathrm{d}, 1 \mathrm{H}, J=10.4 \mathrm{~Hz}), 5.22(\mathrm{~d}, 1 \mathrm{H}, J=20.4 \mathrm{~Hz}), 5.89$ (ddt, $1 \mathrm{H}, J=20.4,10.4,5.0 \mathrm{~Hz}), 6.65(\mathrm{~d}, 2 \mathrm{H}, J=8.0 \mathrm{~Hz}), 7.64(\mathrm{~d}$, $2 \mathrm{H}, J=8.4 \mathrm{~Hz}$ ), 7.84 (brs, $1 \mathrm{H}) \mathrm{ppm}$.

${ }^{13} \mathrm{C}$ NMR $\left(\mathrm{CDCl}_{3}\right) \delta 35.2,40.0,52.0,62.3,111.0,118.7$, $118.9,128.4,133.0,152.7,167.2,173.1 \mathrm{ppm}$.

IR (neat) 3734, 3648, 3283, 2951, 1738, 1608, 1520, 1437,

$1363,1300,1219,921,828,772 \mathrm{~cm}^{-1}$.

$[\alpha]_{\mathrm{D}}^{23}+6.50\left(c 1.44, \mathrm{CHCl}_{3}, 90 \% \mathrm{ee}\right)$.

HRMS: calcd. for $\mathrm{C}_{15} \mathrm{H}_{22} \mathrm{~N}_{3} \mathrm{O}_{3}\left([\mathrm{M}+\mathrm{H}]^{+}\right)$: 292.1656; found: 292.1650 .

HPLC (Daicel Chiralcel OD, Hexane $/ i-\mathrm{PrOH}=4 / 1$, flow rate $=1.0 \mathrm{~mL} / \mathrm{min}$ ) $t_{\mathrm{R}}=13.0$ $\min (R), t_{\mathrm{R}}=17.9 \min (S)$. 
(2R,3R)-Methyl 2-[N'-(p-dimethylaminobenzoyl)hydrazino]-3-methyl-4-pentenoate<smiles>C=CC(C)[C@H](NNC(=O)c1ccc(N(C)C)cc1)C(=O)OC</smiles>

(3i):

${ }^{1} \mathrm{H}$ NMR $\left(\mathrm{CDCl}_{3}\right) \delta 1.15(\mathrm{~d}, 3 \mathrm{H}, J=6.8), 2.64(\mathrm{td}, 1 \mathrm{H}, J=$ 14.8, 7.1 Hz), $3.05(\mathrm{~s}, 6 \mathrm{H}), 3.68(\mathrm{~m}, 1 \mathrm{H}), 3.76(\mathrm{~s}, 3 \mathrm{H}), 5.10$ (brs, $1 \mathrm{H}), 5.14(\mathrm{dd}, 1 \mathrm{H}, J=6.9,1.3 \mathrm{~Hz}), 5.17(\mathrm{dd}, 1 \mathrm{H}, J=$ 12.6, $1.3 \mathrm{~Hz}), 5.86(\mathrm{ddd}, 1 \mathrm{H}, J=17.9,9.6,7.6 \mathrm{~Hz}), 6.64(\mathrm{~d}$, $2 \mathrm{H}, J=8.9 \mathrm{~Hz}), 7.62(\mathrm{~d}, 2 \mathrm{H}, J=8.9 \mathrm{~Hz}), 7.69(\mathrm{~d}, 1 \mathrm{H}, J=5.5$ $\mathrm{Hz}) \mathrm{ppm}$.

${ }^{13} \mathrm{C} \mathrm{NMR}\left(\mathrm{CDCl}_{3}\right) \delta 16.9,39.9,40.0,51.8,67.7,111.0,116.5$, $118.9,128.3,139.1,152.7,167.4,173.0 \mathrm{ppm}$.

IR (neat) 3283, 2952, 1737, 1608, 1520, 1444, 1363, 1298, 1204, 1162, 1063, 946, 827, $768 \mathrm{~cm}^{-1}$.

$[\alpha]_{\mathrm{D}}^{23}-2.20\left(c 1.27, \mathrm{CHCl}_{3}, 88 \%\right.$ ee).

HRMS: calcd. for $\mathrm{C}_{16} \mathrm{H}_{24} \mathrm{~N}_{3} \mathrm{O}_{3}\left([\mathrm{M}+\mathrm{H}]^{+}\right)$: 306.1812; found: 306.1814 .

HPLC (Daicel Chiralcel OD-H, Hexane $/ i-\mathrm{PrOH}=19 / 1$, flow rate $=0.5 \mathrm{~mL} / \mathrm{min}$ ) $t_{\mathrm{R}}=$ $66.3 \min (2 R, 3 R), t_{\mathrm{R}}=77.8 \min (2 S, 3 S)$.

(2R,3R)-Methyl $\quad 2$-[N'-(p-dimethylaminobenzoyl)hydrazino]-3-ethyl-4-pentenoate<smiles>C=C[C@H](CC)[C@H](NNC(=O)c1ccc(N(C)C)cc1)C(=O)OC</smiles>
$(3 \mathbf{j})$ :

${ }^{1} \mathrm{H}$ NMR $\left(\mathrm{CDCl}_{3}\right) \delta 0.95(\mathrm{t}, 3 \mathrm{H}, J=7.2 \mathrm{~Hz}), 1.42-1.49(\mathrm{~m}$, $1 \mathrm{H}), 1.58-1.65(\mathrm{~m}, 1 \mathrm{H}), 2.38-2.43(\mathrm{~m}, 1 \mathrm{H}), 3.02(\mathrm{~s}, 6 \mathrm{H}), 3.76$ $(\mathrm{s}, 3 \mathrm{H}), 5.10$ (brs, $1 \mathrm{H}, J=2.7 \mathrm{~Hz}), 5.15(\mathrm{dd}, 1 \mathrm{H}, J=16.8,1.9$ $\mathrm{Hz}), 5.22(\mathrm{dd}, 1 \mathrm{H}, J=12.6,1.9 \mathrm{~Hz}), 5.75(\mathrm{dt}, 1 \mathrm{H}, J=18.8$, $8.6 \mathrm{~Hz}), 6.65$ (d, 2H, $J=8.9 \mathrm{~Hz}), 7.59$ (brs, 1H), 7.61 (d, 2H, $J=9.0 \mathrm{~Hz}) \mathrm{ppm}$.

${ }^{13} \mathrm{C} \mathrm{NMR}\left(\mathrm{CDCl}_{3}\right) \delta 11.6,24.1,40.1,47.7,51.9,66.7,111.0$, $118.4,118.9,128.3,137.3,152.7,167.3,173.3 \mathrm{ppm}$.

IR (neat) 3303, 2969, 1737, 1637, 1608, 1520, 1460, 1362, 1205, $772 \mathrm{~cm}^{-1}$. $[\alpha]_{\mathrm{D}}^{22}+20.3\left(c 0.66, \mathrm{CHCl}_{3}, 87 \%\right.$ ee $)$.

HRMS: calcd. for $\mathrm{C}_{17} \mathrm{H}_{25} \mathrm{~N}_{3} \mathrm{NaO}_{3}\left([\mathrm{M}+\mathrm{H}]^{+}\right)$: 342.1788 ; found: 342.1787 .

HPLC (Daicel Chiralcel OJ-H, Hexane $/ i-\mathrm{PrOH}=9 / 1$, flow rate $=0.5 \mathrm{~mL} / \mathrm{min}$ ) $t_{\mathrm{R}}=42.3$ $\min (2 R, 3 R), t_{\mathrm{R}}=59.1 \min (2 S, 3 S)$.

(2R,3R)-Methyl 2-[N'-(p-dimethylaminobenzoyl)hydrazino]-3-butyl-4-pentenoate<smiles>C=C[C@H](CCCC)[C@H](NNC(=O)c1ccc(N(C)C)cc1)C(=O)OC</smiles>
(3k):

${ }^{1} \mathrm{H}$ NMR $\left(\mathrm{CDCl}_{3}\right) \delta 0.89(\mathrm{t}, 3 \mathrm{H}, J=6.9 \mathrm{~Hz}), 1.24-1.38(\mathrm{~m}$, $4 \mathrm{H}), 1.40-1.46(\mathrm{~m}, 1 \mathrm{H}), 1.50-1.56(\mathrm{~m}, 1 \mathrm{H}), 2.45-2.50(\mathrm{~m}$, $1 \mathrm{H}), 3.01$ (s, 6H), 3.74 (brs, 1H), 3.76 (s, 3H), 5.12 (brs, 1H), $5.12(\mathrm{dd}, 1 \mathrm{H}, J=18.0,1.4 \mathrm{~Hz}), 5.18(\mathrm{dd}, 1 \mathrm{H}, J=10.2,1.4$ $\mathrm{Hz}), 5.75(\mathrm{dt}, 1 \mathrm{H}, J=19.0,8.6 \mathrm{~Hz}), 6.65(\mathrm{~d}, 2 \mathrm{H}, J=8.9 \mathrm{~Hz})$, $7.62(\mathrm{~d}, 2 \mathrm{H}, J=8.9 \mathrm{~Hz}), 7.71(\mathrm{brs}, 1 \mathrm{H}) \mathrm{ppm}$.

${ }^{13} \mathrm{C}$ NMR $\left(\mathrm{CDCl}_{3}\right) \delta 13.9,22.4,29.2,30.6,40.0,46.0,51.9$, $67.0,111.0,118.1,119.0,128.3,137.7,152.7,167.3,173.2$ ppm.

IR (neat) 3852, 3648, 3299, 2928, 1736, 1608, 1507, 1457, 1362, 1299, $1205 \mathrm{~cm}^{-1}$. $[\alpha]_{\mathrm{D}}^{21}+13.1\left(c 1.06, \mathrm{CHCl}_{3}, 87 \%\right.$ ee $)$.

HRMS: calcd. for $\mathrm{C}_{19} \mathrm{H}_{30} \mathrm{~N}_{3} \mathrm{O}_{3}\left([\mathrm{M}+\mathrm{H}]^{+}\right)$: 348.2282; found: 348.2278 . 
HPLC (Daicel Chiralcel OD-H, Hexane $/ i-\mathrm{PrOH}=9 / 1$, flow rate $=0.5 \mathrm{~mL} / \mathrm{min}$ ) $t_{\mathrm{R}}=$ $42.6 \min (2 R, 3 R), t_{\mathrm{R}}=54.9 \min (2 S, 3 S)$.

$(2 R, 3 R)$-Methyl

2-[N'-(p-dimethylaminobenzoyl)hydrazino]-3-(3-methylbutyl)-4-pentenoate (3I):

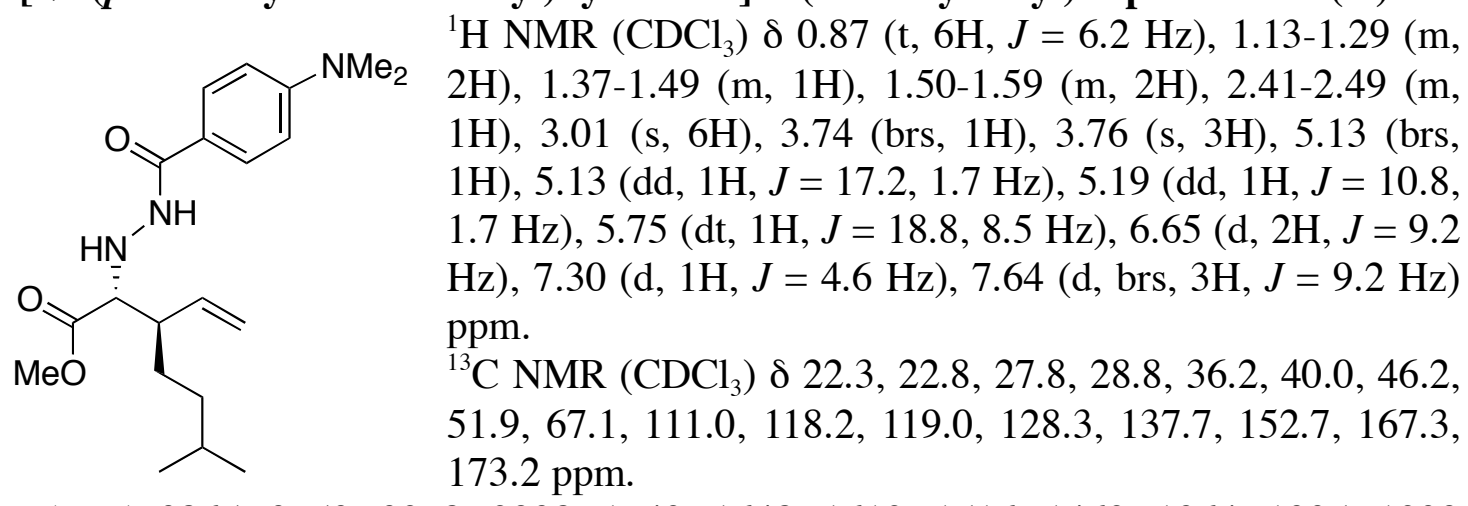

IR (neat) 3865, 3750, 3278, 2938, 1740, 1648, 1612, 1516, 1462, 1364, 1305, 1209 $\mathrm{cm}^{-1}$.

$[\alpha]_{\mathrm{D}}^{21}+3.00\left(c 0.71, \mathrm{CHCl}_{3}, 87 \%\right.$ ee $)$.

HRMS: calcd. for $\mathrm{C}_{20} \mathrm{H}_{32} \mathrm{~N}_{3} \mathrm{O}_{3}\left([\mathrm{M}+\mathrm{H}]^{+}\right)$: 362.2438; found: 362.2427.

HPLC (Daicel Chiralcel OD-H, Hexane $/ i-\mathrm{PrOH}=9 / 1$, flow rate $=1.0 \mathrm{~mL} / \mathrm{min}$ ) $t_{\mathrm{R}}=$ $16.9 \min (2 R, 3 R), t_{\mathrm{R}}=23.2 \min (2 S, 3 S)$.

\section{$(2 R, 3 R)$-Methyl}

2-[N'-(p-dimethylaminobenzoyl)hydrazino]-3-benzyloxy-4-pentenoate (3m):

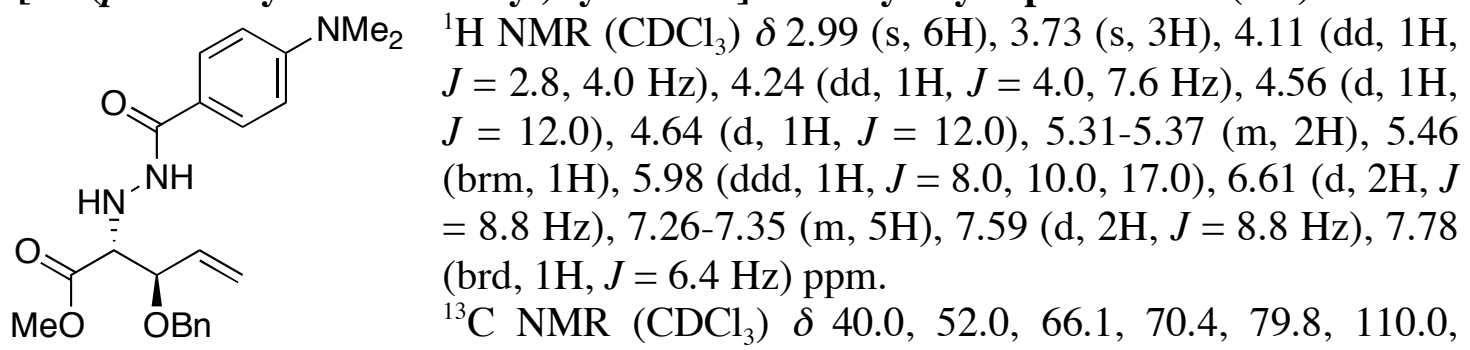

$119.0,120.0,127.5,127.7,128.3,128.3,134.2,137.7,152.6,166.9,171.0 \mathrm{ppm}$.

IR (KBr): 3298, 3257, 3024, 2887, 1739, 1610, 1523, 1346, 1323, 1207, 1159, 1092, 984, 829, 739, 698, $617 \mathrm{~cm}^{-1}$.

$[\alpha]_{\mathrm{D}}^{22}-0.32\left(c 0.53, \mathrm{CHCl}_{3}, 82 \%\right.$ ee $)$.

HRMS: calcd. for $\mathrm{C}_{22} \mathrm{H}_{27} \mathrm{~N}_{3} \mathrm{O}_{4}\left[\mathrm{M}^{+}+\mathrm{Na}\right] 420.1894$, found 420.1888 .

HPLC (Daicel Chiralcel OJ-H, Hexane $/ \mathrm{EtOH}=4 / 1$, flow rate $=1.0 \mathrm{~mL} / \mathrm{min}$ ) $t_{R}=20.6$ $\min (2 R, 3 R), t_{R}=27.0 \min (2 S, 3 S)$.

\section{$\mathrm{Zn}(\mathrm{OH})_{2}$-Catalyzed Asymmetric Allylation:}

$\mathrm{Zn}(\mathrm{OH})_{2}(3.0 \mathrm{mg}, 0.030 \mathrm{mmol})$ was added to water $(3.5 \mathrm{~mL})$, and then the mixture was stirred for $10 \mathrm{~min}$. $\quad \mathbf{4 a}(32.7 \mathrm{mg}, 0.072 \mathrm{mmol})$ and acetone $(7.5 \mathrm{~mL})$ were added and the mixture was stirred for $10 \mathrm{~min}$. After addition of $\mathbf{1 h}(149.7 \mathrm{mg}, 0.60$ $\mathrm{mmol})$, the mixture was stirred at $0{ }^{\circ} \mathrm{C}$ for $15 \mathrm{~min}$, and then $\mathbf{2 a}(92 \mu \mathrm{L}, 0.36 \mathrm{mmol})$ was added. After $36 \mathrm{~h}$, the reaction was quenched with saturated aqueous $\mathrm{NaHCO}_{3}$. The resultant mixture was extracted with $\mathrm{CH}_{2} \mathrm{Cl}_{2}$ (4 times), and the combined organic layers 
were dried over anhydrous $\mathrm{Na}_{2} \mathrm{SO}_{4}$. The solvents were evaporated in vacuo, and the residue was purified by preparative TLC $($ Hexane/AcOEt $=1 / 4)$ to give $\mathbf{3 h}(80 \%$ yield). The enantiomeric excess of the product was determined by HPLC analysis ( $85 \%$ ee).

\section{(R)-Methyl 2-[N-(benzyloxycarbonyl)amino]-4-pentenoate $(6):^{2}$}

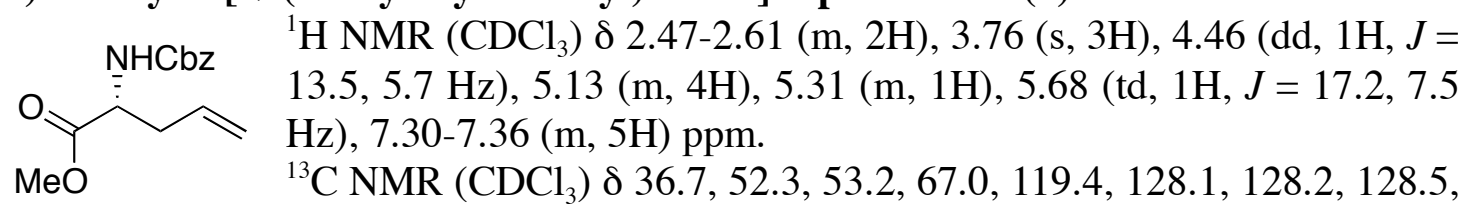

$131.9,136.2,155.7,172.1 \mathrm{ppm}$.

IR (neat) 3861, 3743, 3358, 2948, 1724, 1645, 1516, 1467, 1342, 1216, $1049 \mathrm{~cm}^{-1}$. $[\alpha]_{\mathrm{D}}^{20}-13.7\left(c 0.18, \mathrm{CHCl}_{3}\right)$.

\section{(2R,3R)-Methyl 2-[N-(benzyloxycarbonyl)amino]-3-methyl-4-pentenoate (7): ${ }^{3}$}

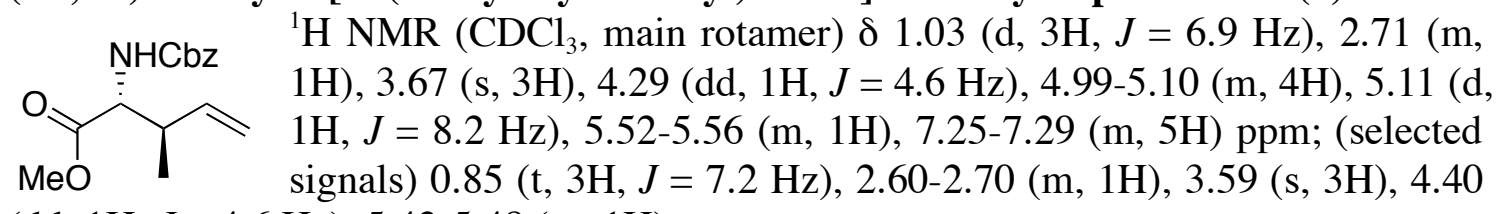
$(\mathrm{dd}, 1 \mathrm{H}, J=4.6 \mathrm{~Hz}), 5.42-5.48(\mathrm{~m}, 1 \mathrm{H}) \mathrm{ppm}$.

${ }^{13} \mathrm{C} \mathrm{NMR}\left(\mathrm{CDCl}_{3}\right.$, main rotamer $) \delta 16.0,40.0,52.2,58.2,67.1,117.0,128.1,128.2$, 128.5, 136.2, 137.5, 156.2, 172.0 ppm; (selected signals) 11.7, 23.5, 48.0, 56.9, 118.4, $136.2 \mathrm{ppm}$.

IR (neat) 3750, 3344, 2960, 1724, 1517, 1449, 1347, 1267, 1216, 1096, 1055, 1017, 931, $771,701 \mathrm{~cm}^{-1}$.

$[\alpha]_{\mathrm{D}}^{21}-8.2\left(c 0.32, \mathrm{CHCl}_{3}\right)$.

(2R,3R)-Methyl 2-[N-(tert-butoxycarbonyl)amino]-3-hydroxy-4-pentanoate $(8) \mathbf{:}^{\mathbf{4}}$

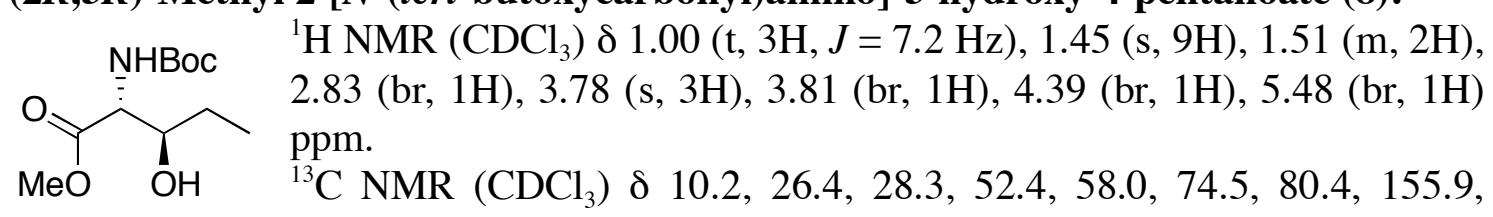
$171.3 \mathrm{ppm}$.

IR (neat): $3435,2977,1714,1505,1165,734 \mathrm{~cm}^{-1}$.

HRMS: calcd. for $\mathrm{C}_{11} \mathrm{H}_{22} \mathrm{NO}_{5}\left[\mathrm{M}^{+}+\mathrm{H}\right]$ 248.1492, found 248.1496.

$[\alpha]_{\mathrm{D}}^{22}=-15.1\left(c 1.6, \mathrm{CHCl}_{3}\right)$.

\section{NMR Experiments for Allylzinc Species:}

When hydrazono ester $\mathbf{1 g}$ was treated with allylboronate $\mathbf{2 a}$ in the presence $\mathrm{ZnF}_{2}$ $(10 \mathrm{~mol} \%)$ and chiral diamine $4 \mathbf{a}(12 \mathrm{~mol} \%)$ in $\mathrm{D}_{2} \mathrm{O} /$ acetone- $_{6}(3 / 5)$ at $0.025 \mathrm{M}$ at room temperature, a new signal at $48.3 \mathrm{ppm}$ in ${ }^{13} \mathrm{C}$ NMR spectrum was observed. On the other hand, a similar signal at $48.1 \mathrm{ppm}$ in ${ }^{13} \mathrm{C} \mathrm{NMR}$ was observed when allylbromide and $\mathrm{Zn}$ (1.0 equiv) were combined in sat. $\mathrm{NH}_{4} \mathrm{Cl}$ aq./acetone- $\mathrm{d}_{6}(3 / 5)$ at room temperature. The signals were assigned as the methylene carbons of allylzinc species. We performed similar experiments for $\alpha$-methyl-substituted allylborate $\mathbf{2 f}$, however, they have so far proved unsuccessful. 


\section{Determination of the Absolute Configuration of Compound 3m}

The absolute configuration of compound $\mathbf{3 m}$ was assigned by transformation into the literature known compound $\mathbf{8}$ as shown in Scheme 2 (text). Compared with our measurement of the optical rotation of $\mathbf{8}$, we found that $[\alpha]_{\mathrm{D}}{ }^{22}$ value was -15.1 (c 1.6, $\mathrm{CHCl}_{3}$ ). Thus, we first assigned that the absolute configuration was $2 S, 3 S$ by comparison of our value with that reported in the literature $\left([\alpha]_{\mathrm{D}}{ }^{25}=-6.9\right.$ (c 2.0, $\left.\left.\mathrm{CHCl}_{3}\right)\right){ }^{4}$ In order to obtain further evidence, we also transformed $\mathbf{8}$ into $\mathbf{9}^{5}$ according to Scheme S1.

Scheme S1.

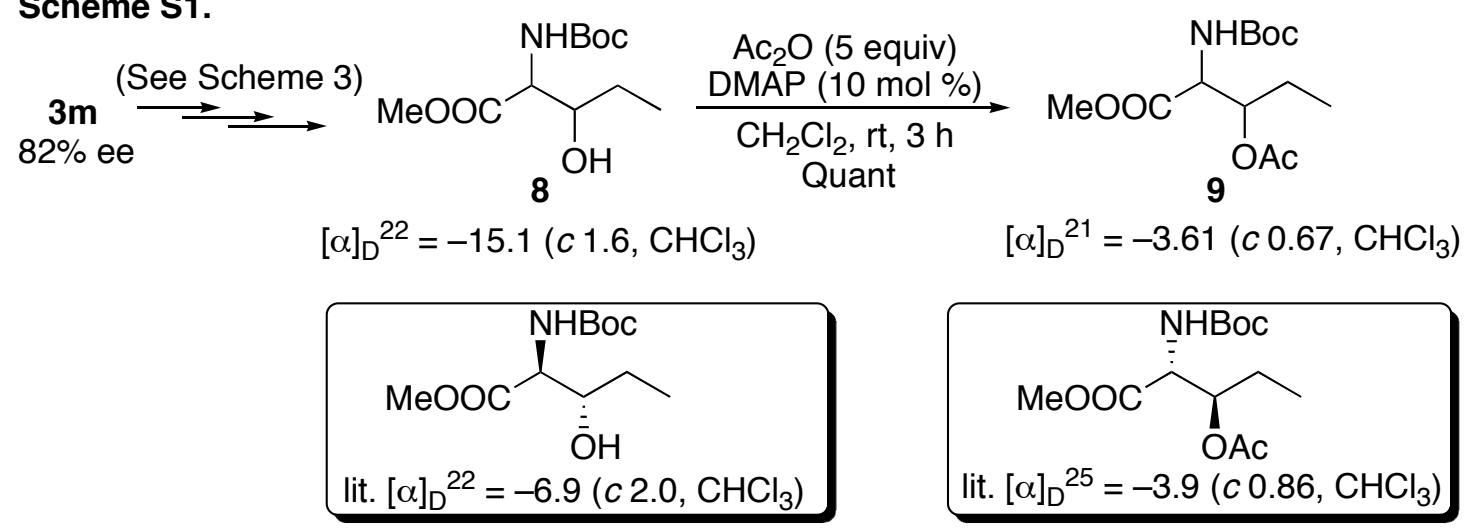

Surprisingly, the result of our measurement was contrary to our previous assignment mentioned above. The optical rotation of compound 9 supported $(2 R, 3 R)$-configuration that is more reliable because of the fitness of these values. We suspected that the literature value of $(2 S, 3 S)-8$ was incorrect. To clear this point, we prepared $(2 S, 3 S)-\mathbf{8}$ from propionaldehyde according to the literature (Scheme S2). ${ }^{4}$ The $[\alpha]_{\mathrm{D}}$ value of $(2 S, 3 S)-8$ prepared by us was found to be +22.4 instead of -6.9 . Thus, we conclude that the literature value was incorrect and that the absolute configuration of $\mathbf{8}$ prepared by us was $2 R, 3 R$. 
Scheme S2. Confirmation of the Literature Value of $(2 S, 3 S)-8$.
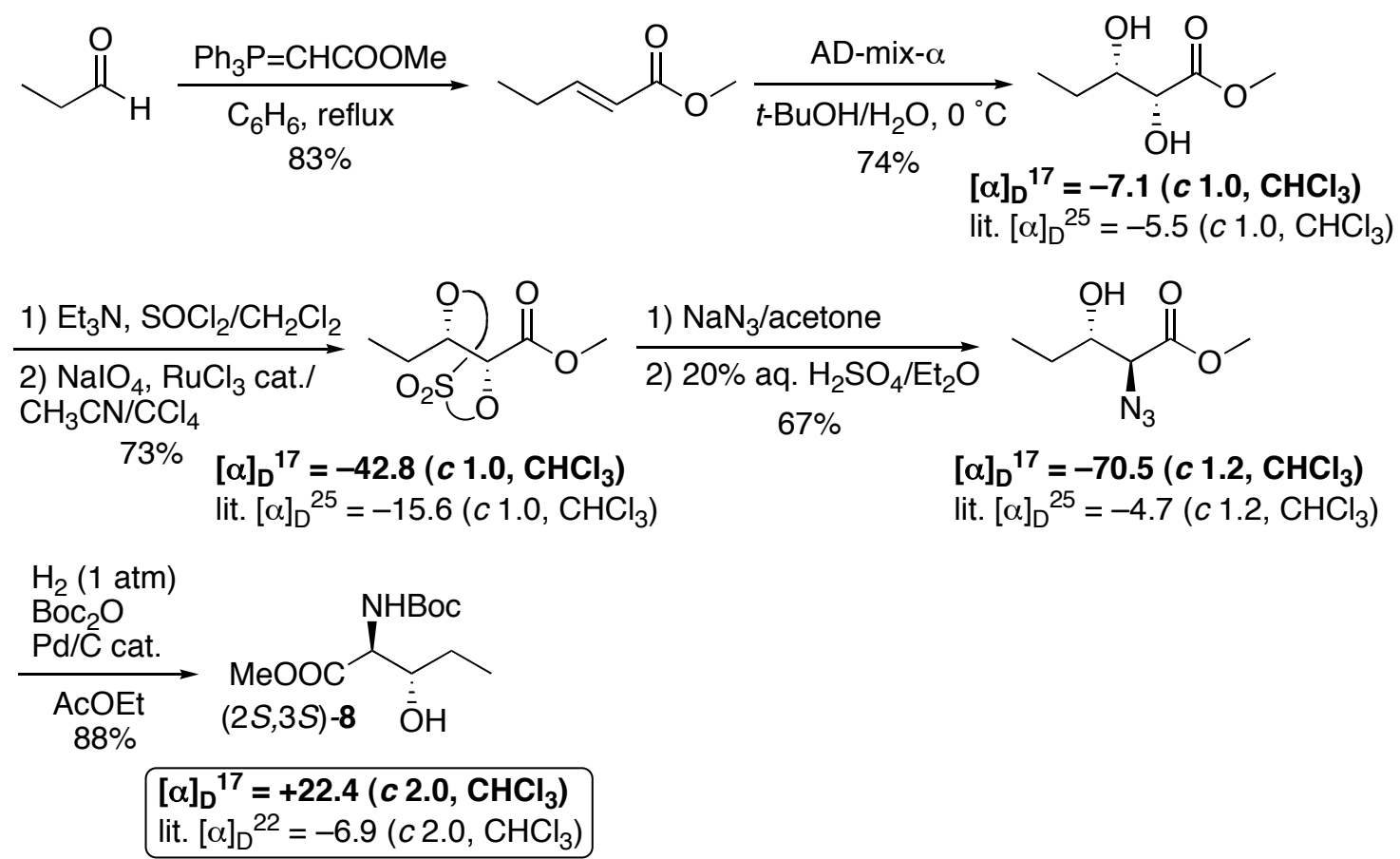

\section{References}

1) Hamada, T.; Manabe, K.; Kobayashi, S. Angew. Chem., Int. Ed. 2003, 42, 3927; Angew. Chem., Int. Ed. 2003, 42, 4565.

2) Abbott, S. D.; Lane-Bell, P.; Sidhu, K. P. S.; Vederas, J. C. J. Am. Chem. Soc. 1994, $116,6513$.

3) Kazmaier, U.; Mues, H.; Krebs, A. Chem.-Eur. J. 2002, 8, 1850.

4) Kandula, S. R. V.; Kumar, P. Tetrahedron: Asymmetry 2005, 16, 3268; delle Monache, G.; Giovanni, M. C. D.; Misiti, D.; Zappia, G. Tetrahedron: Asymmetry 1997, 8, 231.

5) Badorrey, R.; Cativiela, C.; Díaz-de-Villegas, M. D.; Gálvez, J. A. Tetrahedron 1999, $55,14145$. 
$¥ ¥ E c a \neq d a t a ¥ f u j i t a ¥ m-598-1 \mathrm{H} .2$

$\mathrm{m}-598$

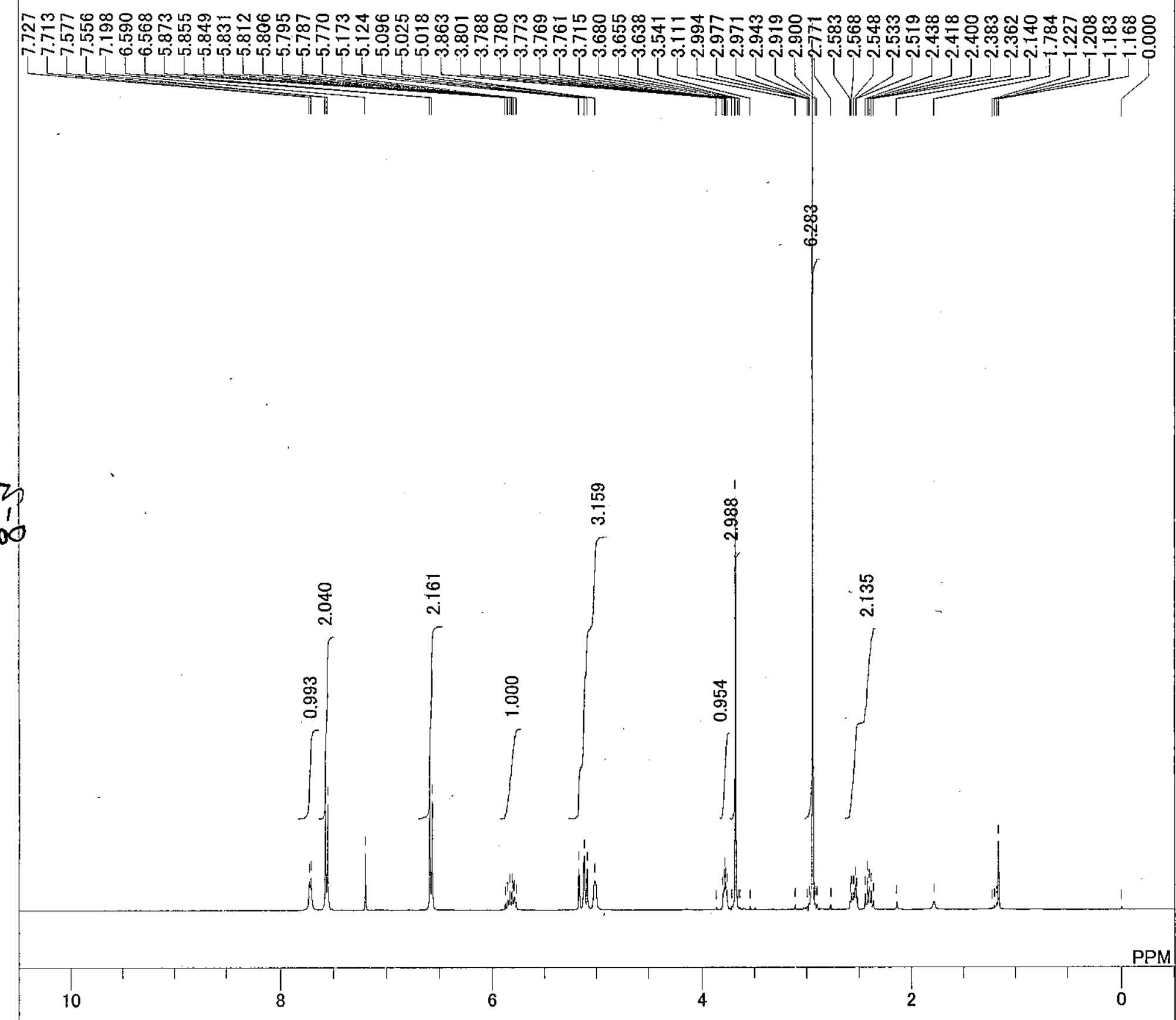

DFILE

COMNT

DATIM

OBNU

XMOD

OBFRQ

OBSET

OBFIN

FREQU

CANS

ACQTM

PW1

RNUC

CTEMP

SLVNT

EXREF

BF

RGAIN

$m-598$

7-03-2007 17:55:14

single_pulse.ex2 9.78 MHz $4.19 \mathrm{KHz}$

$7.29 \mathrm{~Hz}$

16384

$7503.00 \mathrm{~Hz}$

8

$2.1837 \mathrm{sec}$

$2.0000 \mathrm{sec}$

5.50 usec

$1 \mathrm{H}$

CDCL3

$22.7 \mathrm{c}$

$0.00 \mathrm{ppm}$

32

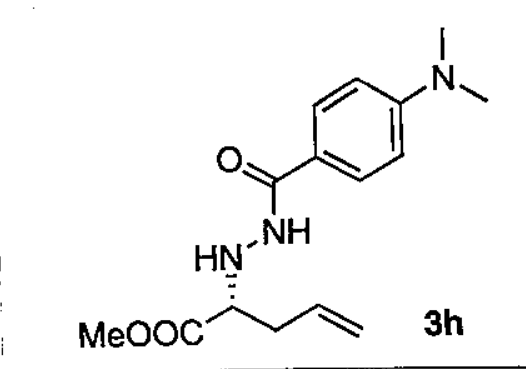




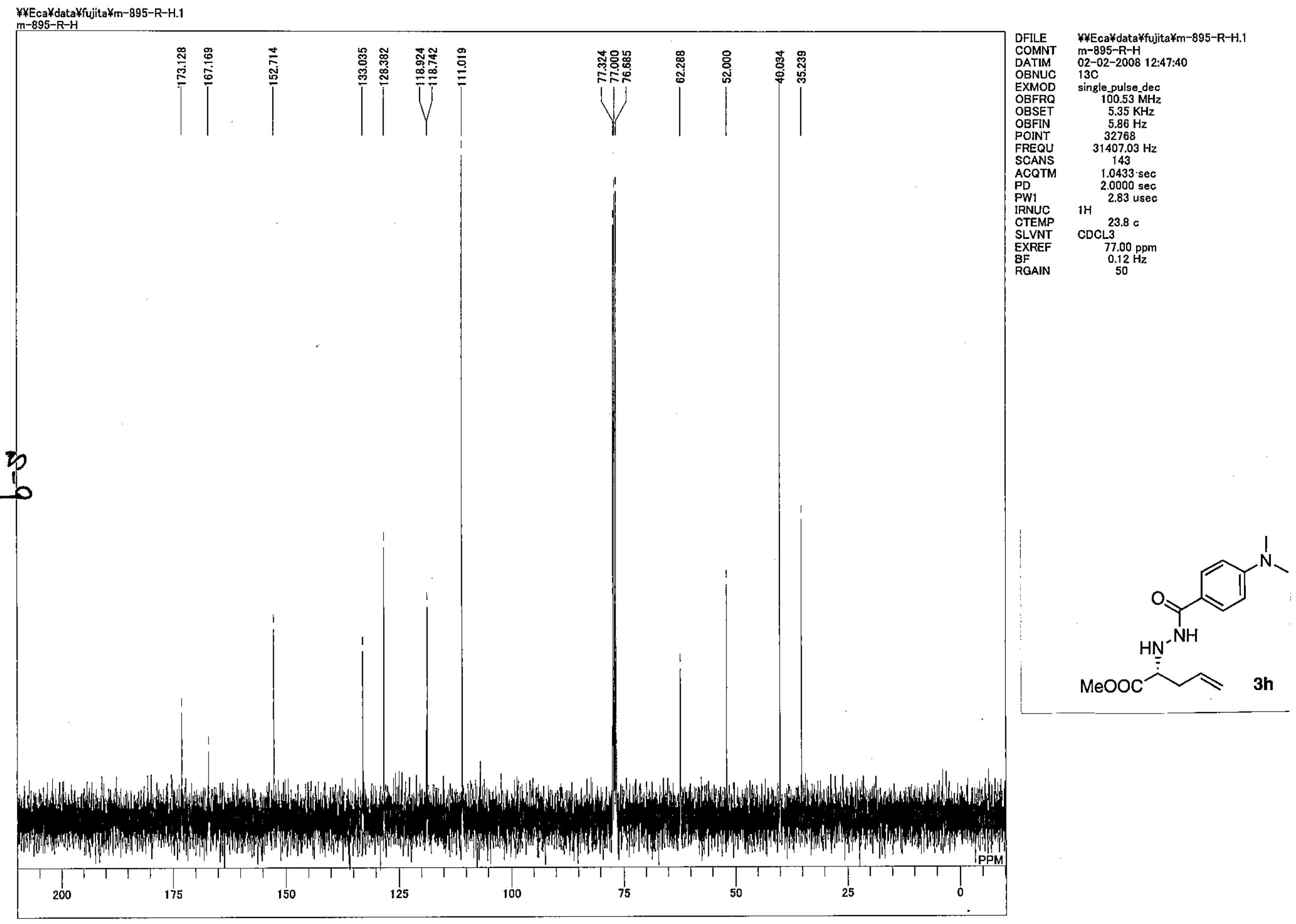


¥¥Eca¥data¥data¥Fujita¥m-663_PROTON.6 m-663

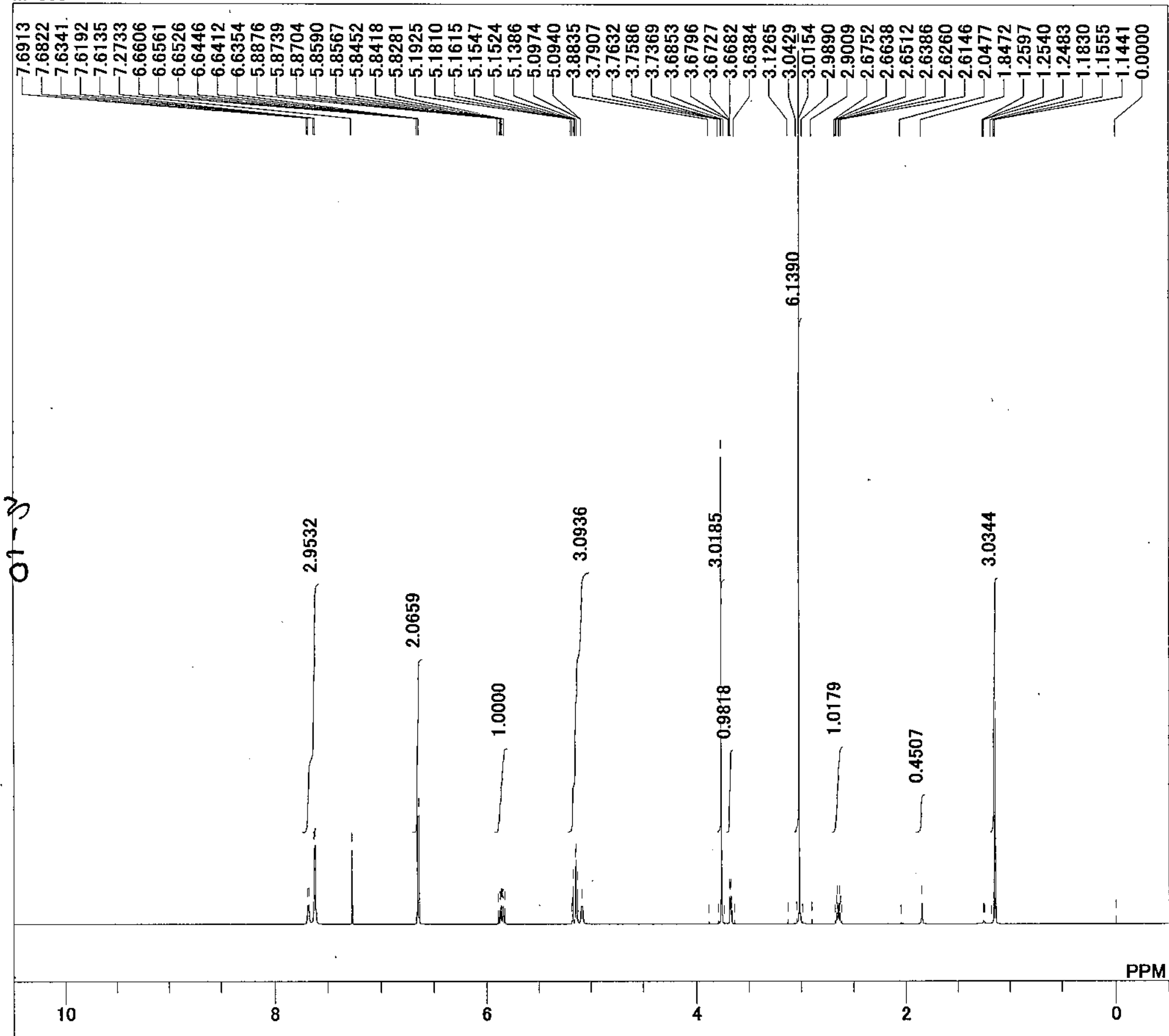

DFILE

COMNT

DATIM

OBNUC

EXMOD

OBFRQ

OBSET

OBFIN

POINT

FREQU

SCANS

ACQTM

PD

PW1

RNUC

CTEMP

SLVNT

EXREF

BF

RGAIN

m-663

27-04

ingle_pulse.ex2 $600.17 \mathrm{MHz}$ $5.30 \mathrm{KHz}$

$5.47 \mathrm{~Hz}$

16384

$11261.26 \mathrm{~Hz}$

$1.4549 \mathrm{sec}$

4.0000 sec

7.30 usec

$1 \mathrm{H}$

CDCL3 19.0

$0.00 \mathrm{ppm}$ $0.12 \mathrm{~Hz}$

40

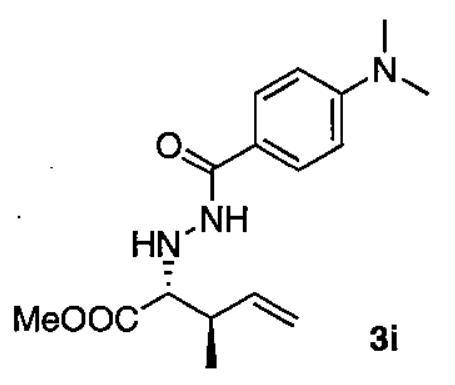


¥¥Eca¥data¥data¥Fujita¥m-663_CARBON.6

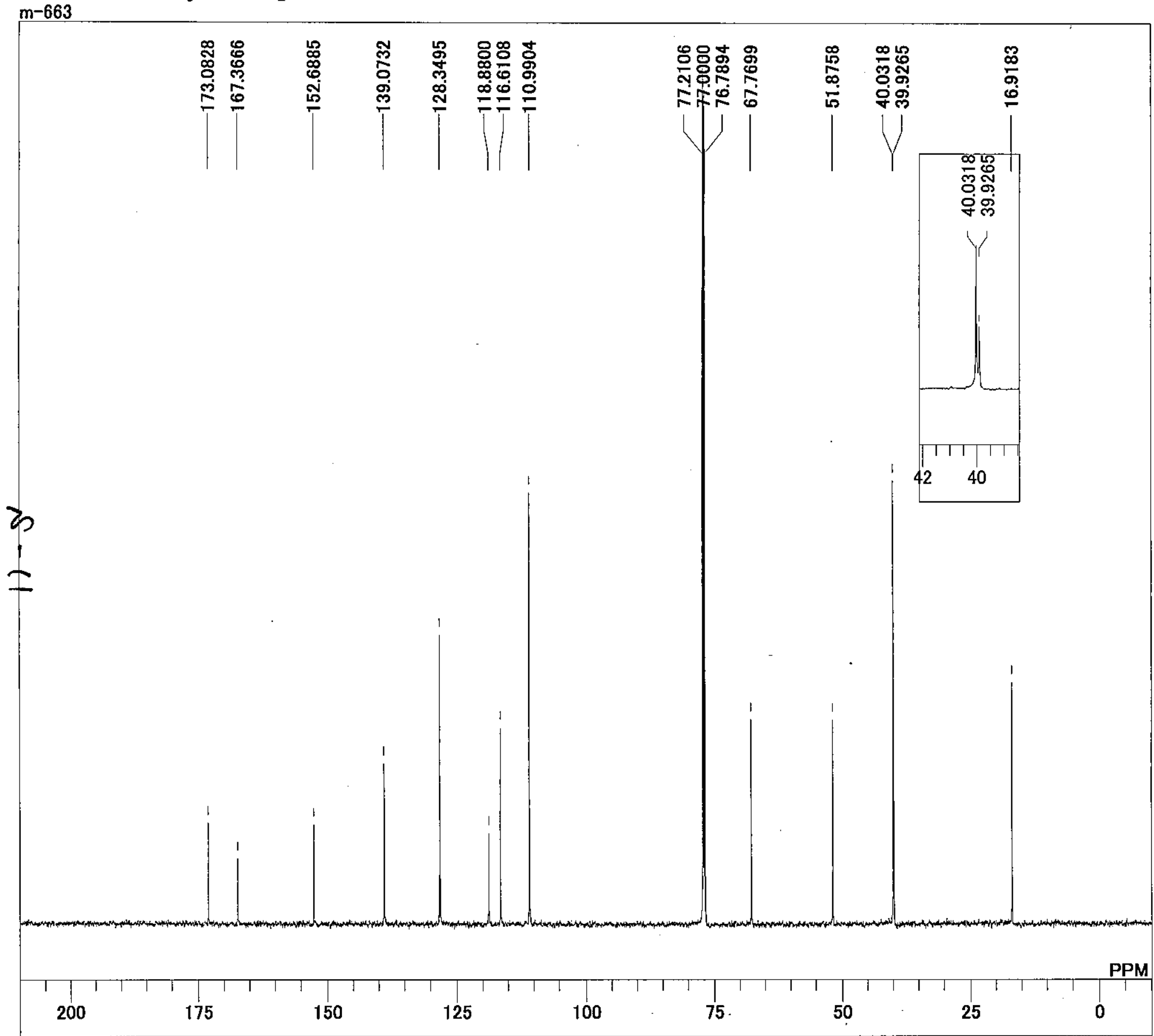

DFILE $¥ ¥ E c a ¥ d a t a ¥ d a t a ¥ F u j i t a ¥ m-663 \_C A R B C$

COMNT $m-663$

DATIM 27-04-2007 10:45:14

OBNUC $13 \mathrm{C}$

EXMOD single_pulse_dec

OBFRQ $\quad 150.92 \mathrm{MHz}$

$\begin{array}{ll}\text { OBSET } & 8.52 \mathrm{KHz}\end{array}$

OBFIN $\quad 1.74 \mathrm{~Hz}$

POINT 32768

FREQU $\quad 47348.49 \mathrm{~Hz}$

SCANS $\quad 1024$

ACQTM $\quad 0.6921 \mathrm{sec}$

PD $2.0000 \mathrm{sec}$

3.45 usec

IRNUC $1 \mathrm{H}$

20.50

SLVNT CDCL3

EXREF $\quad 77.00 \mathrm{ppm}$

$\begin{array}{lc}\text { BF } & 0.12 \mathrm{~Hz} \\ \text { RGAIN } & 60\end{array}$

PPM

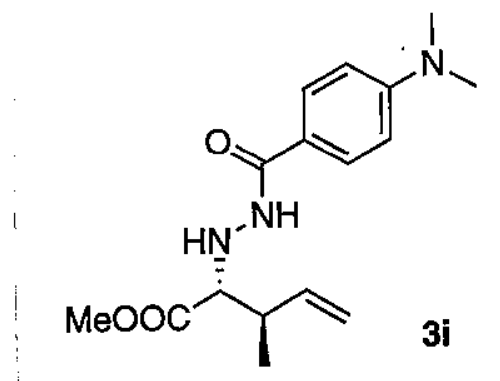




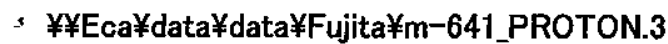

$m-641$

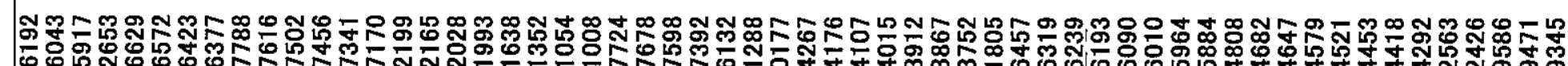

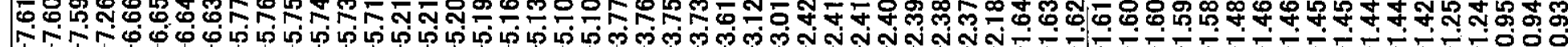

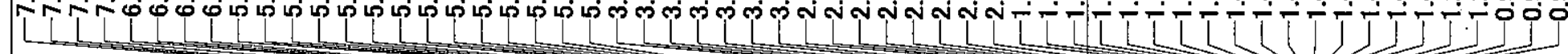

DFILE

COMNT

DATIM

OBNUC

EXMOD

OBFRQ

OBSET

OBFIN

POINT

FREQU

SCANS

ACQTM

PD

PW1

IRNUC

CTEMP

CTEMP

SLVNT

EXREF

BF

RGAIN

$¥ ¥$ Eca $¥$ data $¥$ data $¥ F u j i t a ¥ m-641 \_P R O T C$

$m-641$

07-04-2007 06:29:48

single_pulse.ex2 $600.17 \mathrm{MHz}$ $5.30 \mathrm{KHz}$ $5.47 \mathrm{~Hz}$ 16384 $11261.26 \mathrm{~Hz}$ 16

$1.4549 \mathrm{sec}$

$4.0000 \mathrm{sec}$

$1 \mathrm{H}$

7.30 usec

CDCL3 $19.2 \mathrm{c}$

$0.00 \mathrm{ppm}$

$1.20 \mathrm{~Hz}$

44

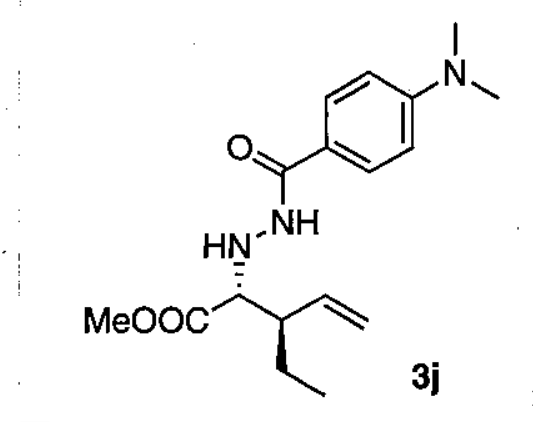

กิ

ֻ

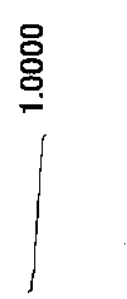

蜡
(1)

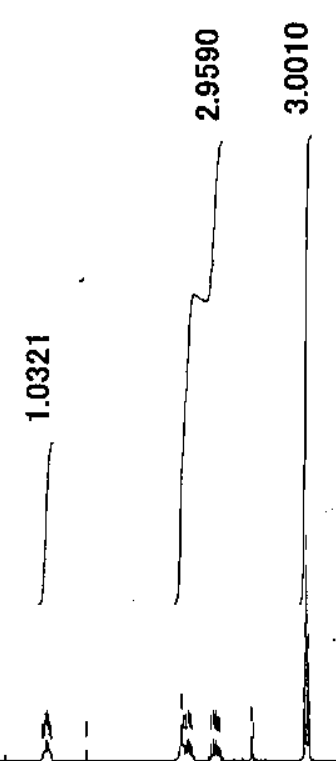

2 
- C:¥Documents and Settings¥All Users 勒ocuments¥Fujita¥m-641_CARBON.als

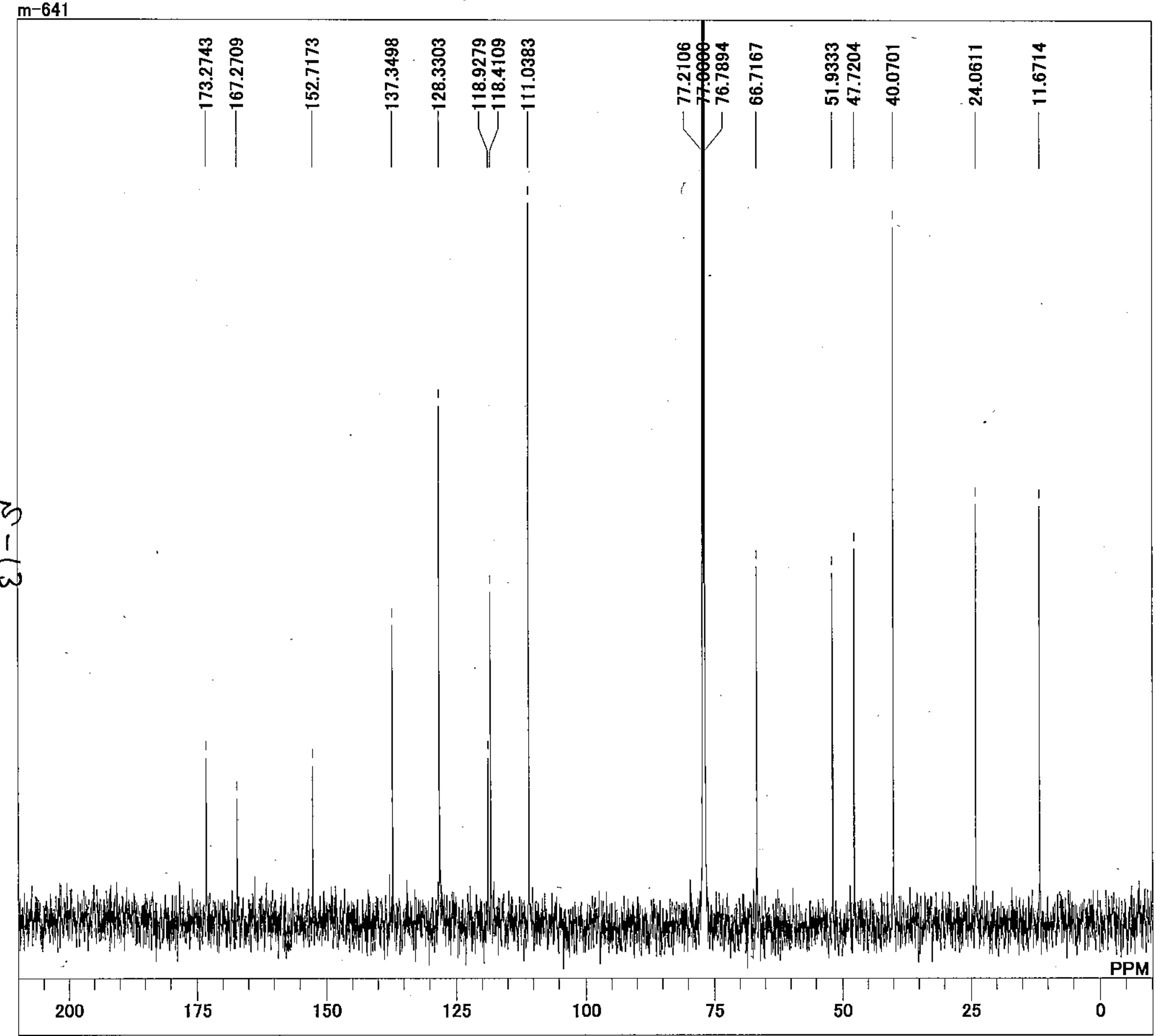

DFILE

COMN

DATIM

OBNUC

EXMOD

OBFRQ

OBSET

POINT

FREQU

SCANS

ACQTM

PD

$2.0000 \mathrm{sec}$

IRNUC $1 \mathrm{H} \quad 3.45$ usec

CTEMP

SLVNT

EXREF

RGAIN

CDCL 3

$77.00 \mathrm{ppm}$

$1.20 \mathrm{~Hz}$

60

gle_pulse_dec $150.92 \mathrm{MHz}$ $8.52 \mathrm{KHz}$ $1.74 \mathrm{~Hz}$ $37878.21 \mathrm{~Hz}$ 512

.6921 sec
C:¥Documents and Settings $¥$ All Users $¥$

06:53:31

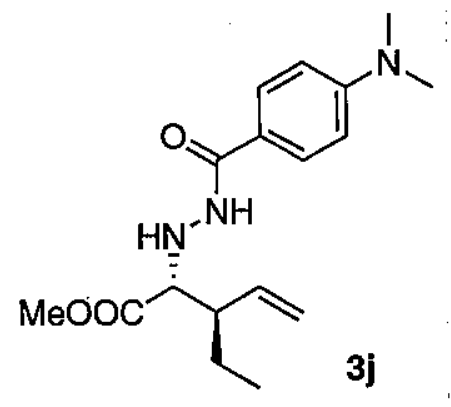


Z:Documents and Settings¥ALCE2¥デスタトップ¥m-924-071019_PROTON.1

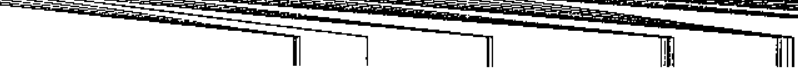

$\frac{1}{A}$

Tiा
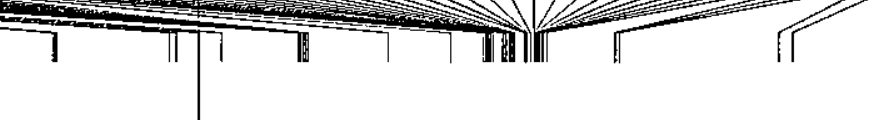

EXMOD

BFRQ

OBSET

POINT

FREQU

SCANS

$\mathrm{PD}$

PW1

IRNUC

CTEMP

EXREF

EXEF

ocuments and Settings:ALCE2¥テテスク 9-10-2007 08:42:48

single.pulse.ex2
$600.17 \mathrm{MHH}$ $600.17 \mathrm{MHz}$
$5.30 \mathrm{KHz}$
$5.47 \mathrm{~Hz}$ 16384

$11261.26 \mathrm{~Hz}$

$11261.26 \mathrm{~Hz}$
16
1.4549

$1.4549 \mathrm{sec}$

6.75 usec

$23.4 \mathrm{c}$

$0.00 \mathrm{ppm}$

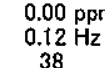

(
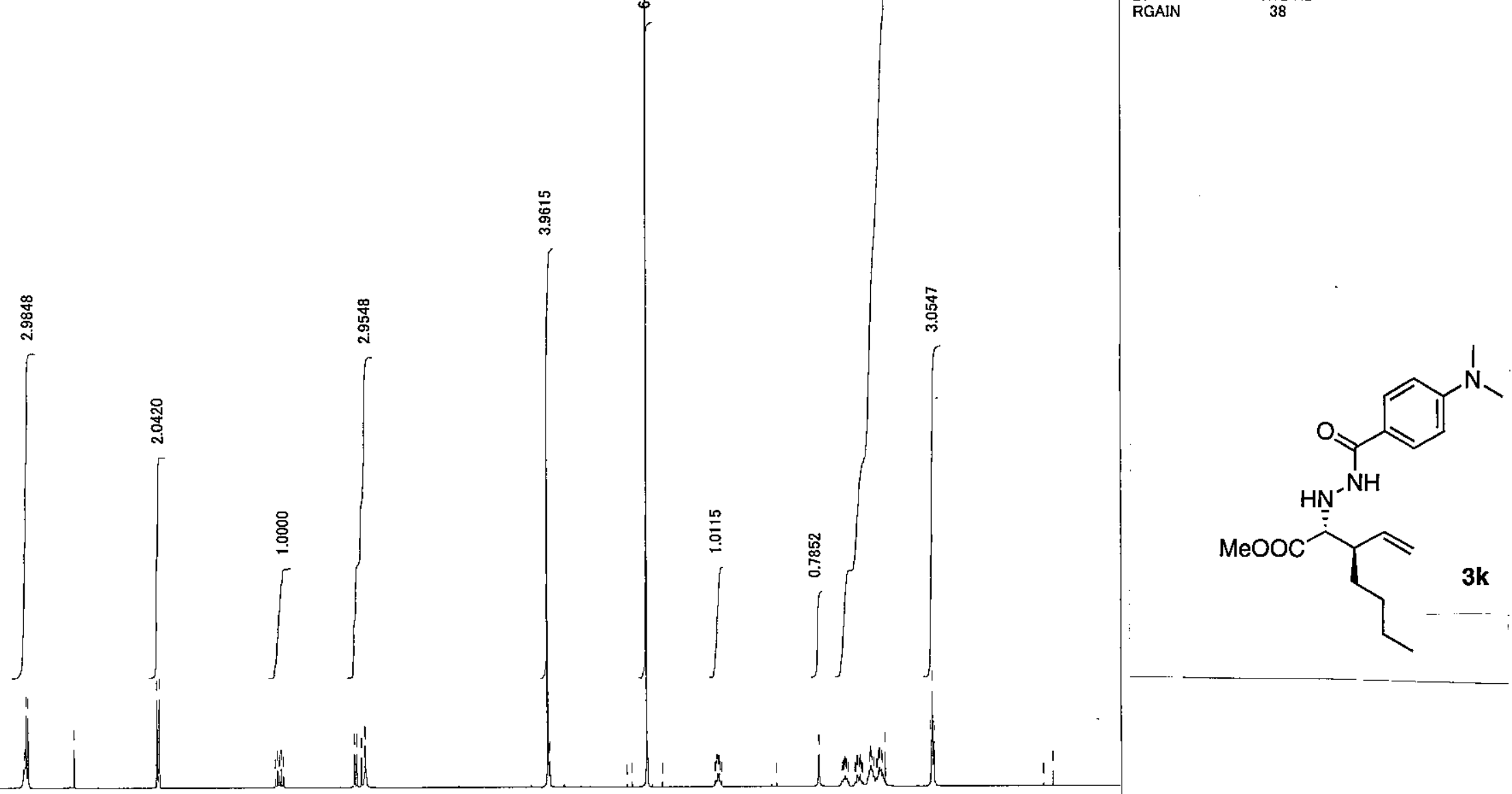

\author{
.
}




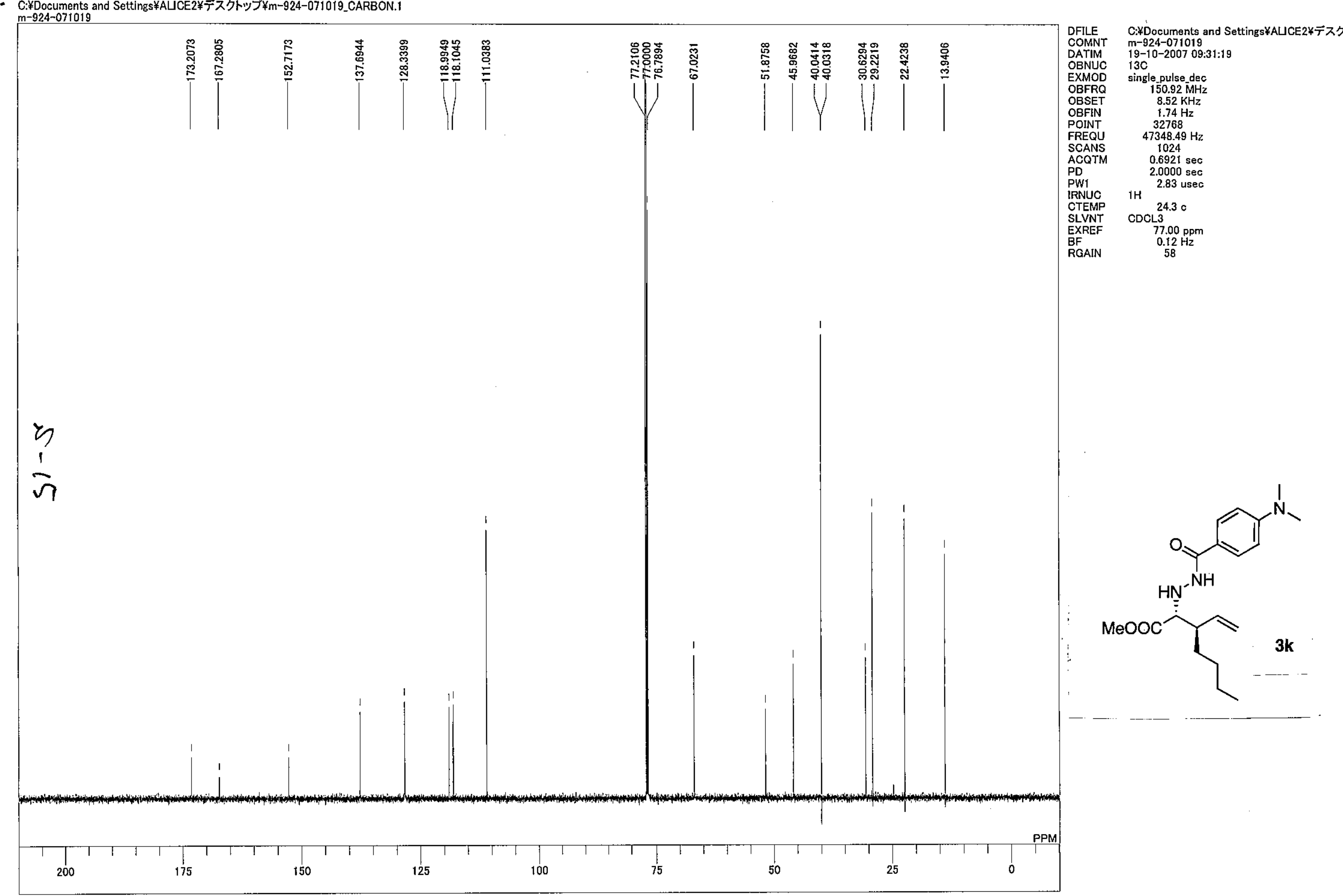




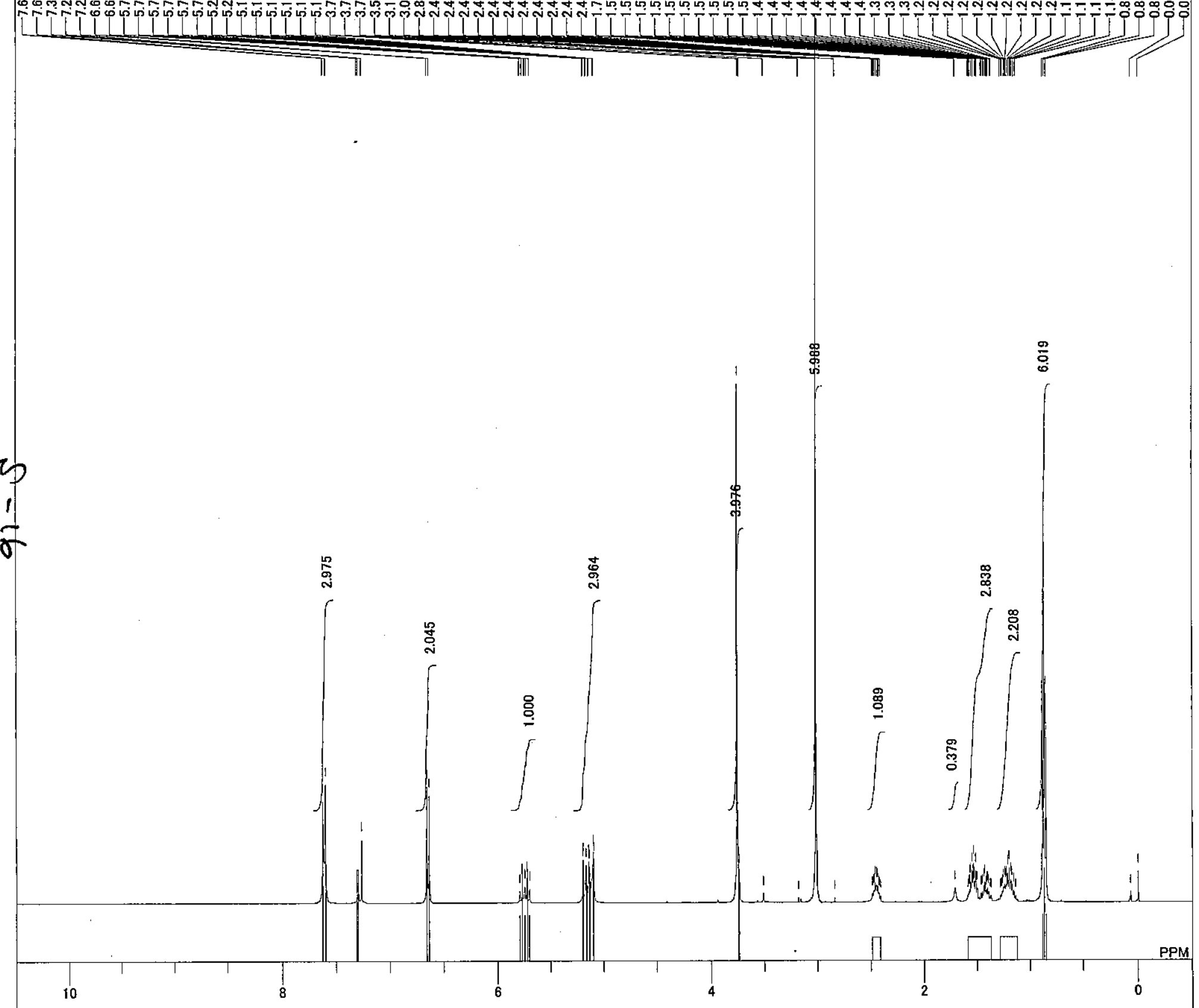

OBFR

OBFIN

FREQU

SCANS

PD

PNUC

CTEMP

SLVNT

EXREF

RGAIN

\&fujita*m-901-1000*m-954.als

16-11-2007 13:39:55

single pulse.ex2

$399.78 \mathrm{MHz}$

$4.19 \mathrm{KHz}$
$7.29 \mathrm{~Hz}$

$7.29 \mathrm{~Hz}$

$7503.00 \mathrm{~Hz}$

8
$2.1837 \mathrm{sec}$

$2.0000 \mathrm{sec}$

$1 \mathrm{H}$

$\operatorname{CDCL}^{22.7}$

$1 \mathrm{H}$-NMR (CDCl3) $\delta$ :

$5.75(1 \mathrm{H}, \mathrm{dt}, \mathrm{J}=18.8,8.5 \mathrm{~Hz})$.

5.16 (3H, ddd, $J=25.1,13.9,1.7 \mathrm{~Hz}$ ),

2..59-2. $37(3 \mathrm{H}, \mathrm{m})$ )

$0.87(6 \mathrm{H}, \mathrm{t}, \mathrm{J}=6.2 \mathrm{~Hz})$

象

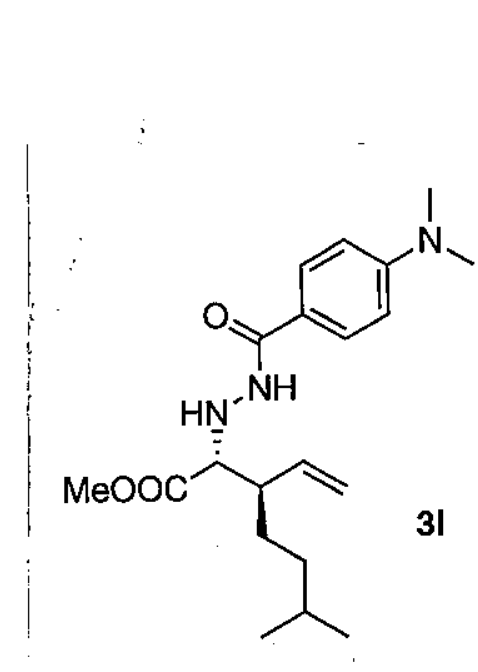




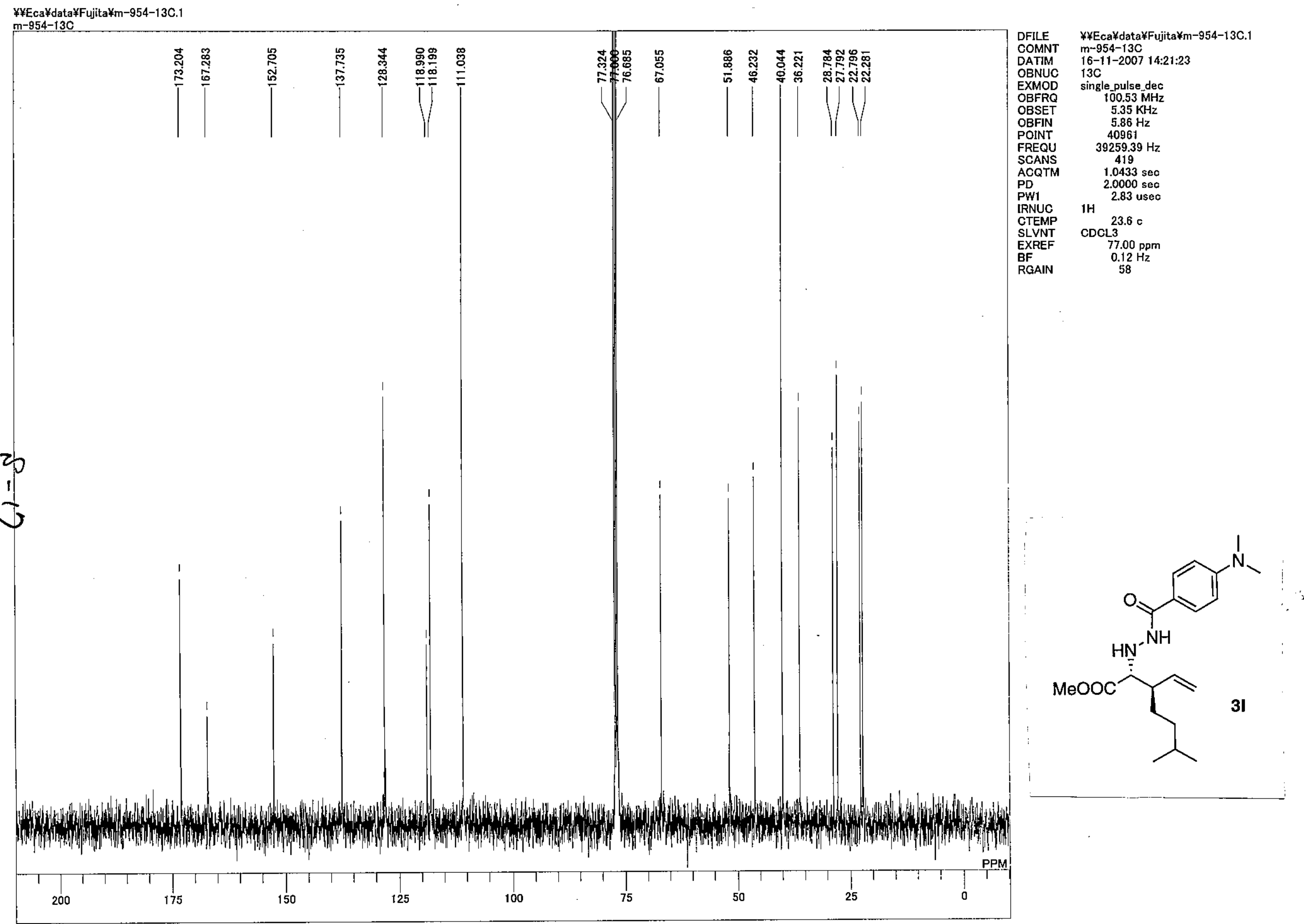


Finca¥data¥nagano¥OBn-re.1

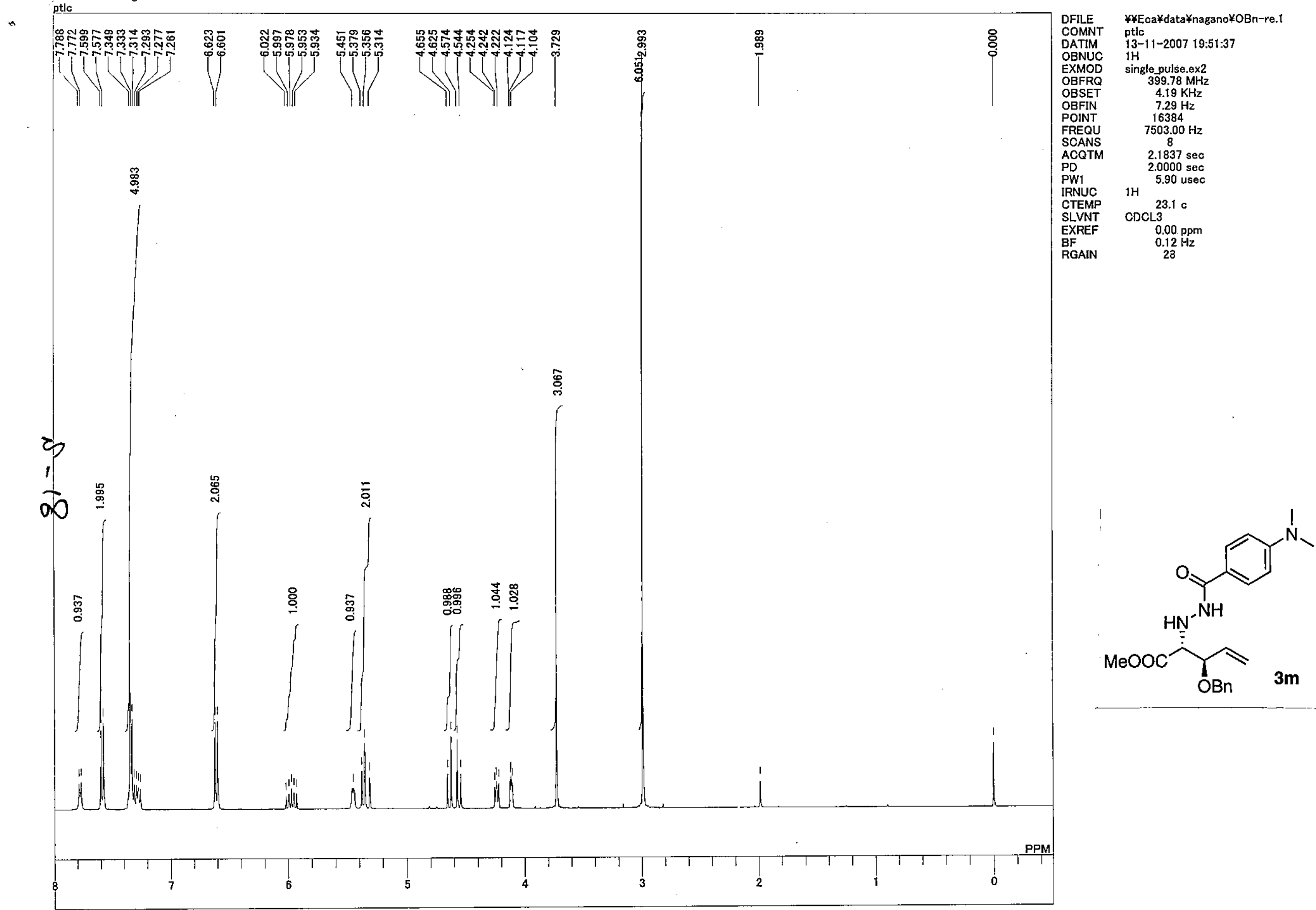


$¥ ¥ E c a ¥ d a t a ¥ n a g a n o ¥ O B n-r e-13 C .1$

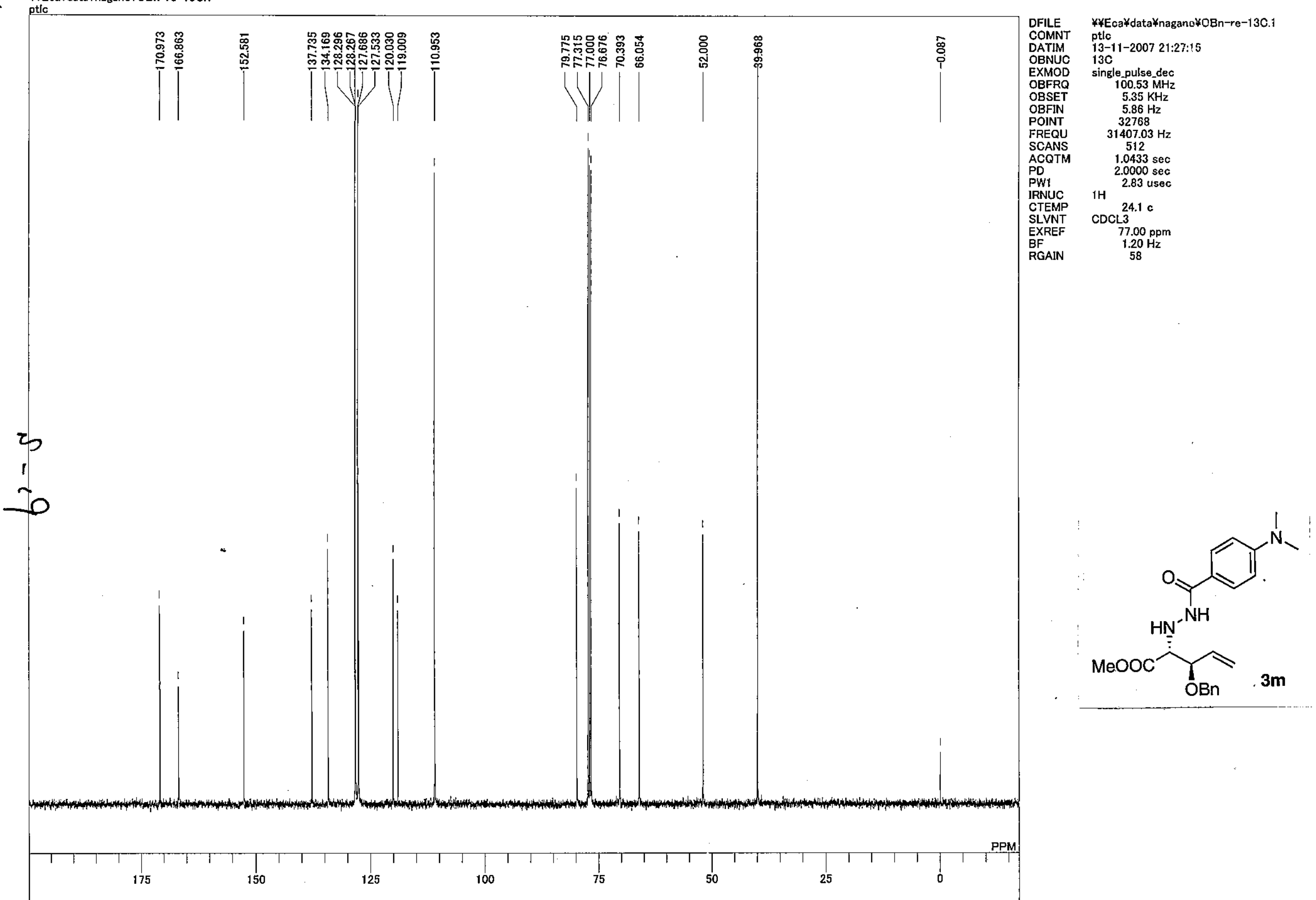

